\title{
A new diagnostic feasibility for cardiomyopathy utilizing acoustic microscopy
}

\author{
Yoichi Nakamura ${ }^{1,2^{*}}$, Kengo Fukushima Kusano ${ }^{3}$, Kazufumi Nakamura ${ }^{3}$, Kazuto Kobayashi ${ }^{4}$, \\ Naohiro Hozumi $^{5}$, Yoshifumi Saijo ${ }^{6}$, Tohru Ohe ${ }^{3,7}$ \\ ${ }^{1}$ Department of Cardiovascular Medicine, Matsuyama Shimin Hospital, Matsuyama, Japan \\ ${ }^{2}$ The Clinic of Soyokaze Cardiovascular Medicine and Diabetes Care, Matsuyama, Japan \\ ${ }^{3}$ Department of Cardiovascular Medicine, Okayama University Graduate School of Medicine, Dentistry and Pharmaceutical Sciences, \\ Okayama, Japan \\ ${ }^{4}$ Department of Research and Development, Honda Electronics Co., Ltd., Toyohashi, Japan \\ ${ }^{5}$ Toyohashi University of Technology, International Cooperation Center for Engineering Education Development, Toyohashi, Japan \\ ${ }^{6}$ Biomedical Imaging Laboratory, Graduate School of Biomedical Engineering, Tohoku University, Sendai, Japan \\ ${ }^{7}$ Department of Cardiology, Sakakibara Heart Institute of Okayama, Okayama, Japan \\ Email: *'y.nakamura@soyokaze-cvd.jp
}

Received 1 November 2012; revised 2 December 2012; accepted 12 December 2012

\begin{abstract}
Aims: Dilated cardiomyopathy often shows left ventricular systolic dysfunction, although histologically it always exhibits non-specific abnormality. We hypothesized that myocyte sound speed might be altered due to incomplete protein accumulation in cells. Methods and Results: Ninety eight biopsied samples were obtained from 49 patients comprising 43 with clinical dilated cardiomyopathy and 6 with hypertrophic cardiomyopathy. Sound speed was evaluated in de-paraffinized $10 \mu \mathrm{m}$ thick sections using an acoustic microscope (frequency range: 50 - $105 \mathrm{MHz}$ ). Conventional histology revealed 7 cases of persistent myocarditis derived from clinical dilated cardiomyopathy samples. Histology of the remaining dilated cardiomyopathy patients indicated non-specific abnormality. All hypertrophic cardiomyopathy cases exhibited myocardial disarray. Ten normal autopsied hearts were compared as controls. The sound speed of controls was $1627 \pm 30 \mathrm{~m} / \mathrm{sec}$. The sound speed in dilated cardiomyopathy samples $(1700 \pm 51 \mathrm{~m} / \mathrm{sec})$ was 1.045-fold faster compared to controls. The sound speed in hypertrophic cardiomyopathy samples (1734 $\pm 51 \mathrm{~m} / \mathrm{sec}, 1.066$-fold compared to controls) was faster than that of the myocarditis group $(1672 \pm 30$ $\mathrm{m} / \mathrm{sec}, \mathbf{1 . 0 2 8}$-fold $)(P=\mathbf{0 . 0 2 1 8})$. Furtheremore, desmin expression was evaluated as extent of emergence (grading 0 - 4). The desmin expression score in hypertrophic cardiomyopathy samples $(2.7 \pm 0.8)$ was significantly higher than in other groups (dilated 2.0 \pm 1.4 , myocarditis $1.6 \pm 1.5$ vs, controls $0, P \leq 0.0001$, 0.0001, 0.0129, respectively). Conclusion: Cardio-
\end{abstract}

${ }^{*}$ Corresponding author. myopathy enhanced the sound speed, which correlated with the elasticity of myocytes, following the impaired compliance of left ventricle, despite the absence of histological changes. The elevation of sound speed of myocytes may be linked to cytoskeletal changes. Myocyte sound speed may be a new diagnostic tool for diagnosis of idiopathic cardiomyopathy independently of conventional histological diagnosis.

Keywords: Cardiomyopathy; Acoustic Microscopy; Heart Failure; Myocytes; Sound Speed; Diagnosis

\section{INTRODUCTION}

Genetic cardiomyopathy is one of the causes of systolic and diastolic heart failure based on abnormality of proteins which comprise intra-myocyte architecture. Consequently, myocytes often contribute to several grades of hypertrophy with attenuation which occasionally advances to replacement fibrosis. Degeneration of myocytes with/without fibrosis leads to a diminished ejection performance and reduced ventricular elasticity. In the last decade, developments in molecular technology have allowed characterization of many gene mutations related to cardiomyopathy. However, the mutation has been identified in just $20 \%-48 \%$ of dilated cardiomyopathy cases, despite identification of over six hundred dissimilar mutation points. Even hypertrophic cardiomyopathy is not confirmed as a gene mutation in all cases [1-4]. In addition, because cardiomyopathies exhibit several phenotypes and a varied pathogenesis with few characteristic findings, it is quite difficult to obtain a precise diagnosis. For this reason, the diagnosis of cardiomyopathy "is now confused", although some Societies have made a statement 
concerning the classification of cardiomyopathies $[5,6]$.

Cardiac biopsy is an effective diagnostic tool for identification of myocardial diseases. Some Societies have recommended a role for endomyocardial biopsy in a number of clinical scenarios [7]. Endomyocardial biopsy is associated with low sensitivity and high specificity because suspected lesions of acquired cardiomyopathy are always scattered over the ventricular wall, leading to sampling errors $[8,9]$; conventional histology then reveals that idiopathic cardiomyopathy demonstrates nonspecific abnormal findings, despite visualization of large numbers of myocytes [10].

Cardiomyopathy, of which there are several grades, exhibits diastolic dysfunction resulting from low compliance, decreased distensibility and viscoelasticity. Consequently the left ventricle exhibits increased stiffness, prolonged relaxation time; thereafter the tau is extended. These conditions result from cardiac overload, heterogeneity of ventricle, over-handling of calcium by myocytes etc. We speculated whether myocytes had an abnormal intra-cellular architecture with/without calcium over-handling, as a result of which ventricular elasticity was also impaired.

Acoustic microscopy has been used to visualize tissue elasticity. This technology was developed by Sokolov [11] in 1949, from which Quate and Lemons [12] further developed the scanning acoustic microscope, the so called SAM in 1975. Nowadays, it is utilized in several clinical and biological fields even as paraffin-embedded samples [13-17].

We hypothesized that if cardiomyopathy had altered the intra-cellular component, its elasticity might change. To clarify changes in cell elasticity we measured the sound speed of myocytes using the SAM system. Acquired cardiomyopathy may be associated with less change in intra-cellular architecture because basic cell architecture of the original cells ought to be comprised of normal cell components; however, focal necrosis and fibrosis were present, due to 1) inflammation caused by virus infection or disturbance of the autoimmune systems, 2) attack by toxins, or 3) abnormal metabolite deposition etc. Thus some idiopathic cardiomyopathies could be readily differentiated from other acquired cardiomyopathies using a parameter such as "cell sound speeds", whereas such differentiation cannot be achieved histologically.

\section{METHODS}

\subsection{Subjects}

Patients diagnosed with congestive heart failure of unknown etiology underwent cardiac biopsy from rightsided ventricular septum to characterize the pathogenesis.
A total of 98 biopsy samples were obtained from 49 congestive heart failure patients who had experienced systolic dysfunction or who were diagnosed with diastolic heart failure.

Samples were registered from two separate Centers, namely the Department of Cardiovascular Medicine of Okayama University Graduate School of Medicine, Dentistry and Pharmaceutical Sciences, and the Department of Cardiovascular Medicine of Matsuyama Shimin Hospital. Congestive heart failure patients with ischemic cardiomyopathy, valvular heart diseases, congenital structural heart diseases, or secondary cardiomyopathy were excluded from this study. The study protocol was approved by the Human Ethics Review Committee of each hospital and a signed consent form was obtained from every patient, or their family if autopsied samples were used. Ten autopsied normal hearts selected from cases of non-cardiac death were compared as controls.

\subsection{Catheterization and Ventriculography}

All patients underwent left and right ventriculography after pressure investigation in both ventricles. Thereafter endomyocardial biopsy samples were obtained from right ventricular septum via the right jugular vein. Left ventricular volume and ejection fraction were calculated by the centerline method.

\subsection{Preparation of Materials}

All specimens were obtained from biopsy or autopsied samples. All samples were fixed with $10 \%$ formaldehyde. Thereafter, specimens were dehydrated with ethanol according to standard processing for histology and embedded in paraffin. Continuous de-paraffinized $5 \mu \mathrm{m}$ thick sections were prepared for standard histological evaluation together with hematoxylin and eosin staining and Masson trichrome staining, and further prepared for immunohistological evaluation. Consecutive $10 \mu \mathrm{m}$ thick specimens sliced together with the $5 \mu \mathrm{m}$ thick sections were also prepared for acoustic microscopy.

\subsection{Acoustic Microscopy $[15,16]$}

An acoustic wave of $5 \mathrm{~ns}$ pulse width with a $10 \mathrm{KHz}$ repetition rate was irradiated and received by the same transducer. The transducer was $1.2 \mathrm{~mm}$ in aperture diameter, and $1.5 \mathrm{~mm}$ in focal length. Its nominal frequency range was $50-105 \mathrm{MHz}(-6 \mathrm{~dB})$, with the central frequency being $80 \mathrm{MHz}$. Distilled water was used for the coupling medium between the specimen and the transducer. Reflections on both sides of the tissue were compared to measure the sound speed and thickness. Two-dimensional profiles of reflection intensity, thickness and sound speed were obtained by mechanically 
scanning the transducer. The transducer was mounted on an X-Y stage driven by the computer. Considering the focal distance and the cross sectional area of the transducer, the diameter of the focal spot was estimated as 20 $\mu \mathrm{m}$ at $80 \mathrm{MHz}$. However, microscopic resolution had been $15 \mu \mathrm{m}$ in the axial plane resolution and $18 \mu \mathrm{m}$ in the lateral plane resolution from the phantom examination. The tissue surface of $2.4 \times 2.4 \mathrm{~mm}$ was covered with 300 by 300 pixels.

\subsection{Measurement of the Sound Speed of Myocytes}

De-paraffinized $10 \mu \mathrm{m}$ thick sections were evaluated using acoustic microscopy. Several specimens were evaluated per patient. The sound speeds of 15 myocytes randomly selected per specimen were measured. The sound speeds of myocytes associated with several specimens per patient were averaged.

\subsection{Immunohistochemistry}

After de-paraffinization, desmin was detected by standard labelled streptavidin biotin methods with a monoclonal anti-human desmin mouse antiserum (Dako Cytomation, Kyoto, Japan) as the primary antibody and biotinylated mouse/rabbit immunoglobulin pig anti-serum (Dako Cytomation, Kyoto, Japan) as the secondary antiserum. Primary antibody was diluted 1:50 and incubated with sections for 90 minutes. The second antiserum was incubated for 30 minutes; thereafter peroxidaseconjugated streptavidin (Dako Cytomation, Kyoto, Japan) was incubated with sections for 30 minutes at room temperature. Finally, the sections were counterstained with hematoxylin.

\subsection{Desmin Expression Score}

Desmin expression score in myocytes of specimens was defined as follows: 0 ; none, 1 ; less than $5 \%, 2 ; 5 \%$ to $25 \%, 3 ; 25 \%$ to $50 \%$ and 4 ; over $50 \%$.

\subsection{Statistics}

All data were expressed as mean \pm standard deviation (SD). Statistical significance of differences among groups was evaluated using ANOVA for continuous variables. A P-value of less than 0.05 was interpreted as statistically significant.

\section{RESULTS}

\subsection{Histology}

Histological evaluation of clinical dilated cardiomyopathy cases showed division into two groups: persistent myocarditis $(\mathrm{n}=7)$ and idiopathic dilated cardiomyopathy $(\mathrm{n}=36)$. All in the persistent myocarditis group exhibited inflammatory cells infiltrated into the interstitium without necrosis of myocytes. Histologically, the dilated cardiomyopathy group showed non-specific abnormality, i.e. 8 almost normal findings, 16 attenuations, 8 mildly increased interstitial fibrosis, 3 scant replacemant fibrosis and one desmin myopathy. The hypertrophic cardio-myopathy group demonstrated typical disarray with myocardial hypertrophy. No materials accumulated in the tissue (Table 1). The control group showed clearly normal findings on the light microscopic image.

\subsection{Clinical Characteristics}

Six patients in the dilated cardiomyopathy group had a family history of heart disease. Clinical findings regarding heart failure in groups are summarized in Table 2. The stage of heart failure (NYHA classification) showed no significant difference among groups. Left ventricular ejection fraction in the dilated cariomyopathy group and the persistent myocarditis group was diminished. Left ventricular end-diastolic pressure in the hypertrophic cardiomyopathy and persistent myocarditis groups was elevated. Pulmonary capillary wedge pressure in the hypertrophic cardiomyopathy group was highest compared to other groups. Brain natriuretic peptide values showed no significant difference among groups despite differences in clinical features.

\subsection{Sound Speed of Myocytes}

Representative images for each group are shown in Figure 1. The sound speed of myocytes in the control group was uniform, as indicated by being within the "green range". The average sound speed was $1627 \pm 30 \mathrm{~m} / \mathrm{sec}$.

The sound speed in the dilated cardiomyopathy group was $1700 \pm 51 \mathrm{~m} / \mathrm{sec}$, which was significantly faster than that of the control group $(\mathrm{P}<0.0001)$. This was 1.045fold compared to controls. Almost all cells are indicated

Table 1. Histological evaluation of clinically diagnosed dilated or hypertrophic cardiomyopathy.

\begin{tabular}{ccc}
\hline Pathological Findings & $\mathrm{n}$ & Group \\
\hline Almost Normal & 8 & \\
Attenuation & 16 & DCM \\
Mildly Increased Interstitial Fibrosis & 8 & \\
Replacement Fibrosis(scant) & 3 & \\
Desmin Myopathy & 1 & HCM \\
Persistent Myocarditis & 7 & Persistent Myocarditis \\
Disarray with Myocardial Hypertropy & 6 & H \\
\hline
\end{tabular}

DCM; dilated cardiomyopathy, HCM; hypertrophic cardiomyopathy. 
Table 2. Clinical characteristics of groups.

\begin{tabular}{|c|c|c|c|c|}
\hline & $\operatorname{DCM}(36)$ & $\operatorname{HCM}(6)$ & Persistent myocarditis (7) & Control (10) \\
\hline Sample number & 79 & 19 & 21 & 10 \\
\hline Age (years) & $57 \pm 15$ & $59 \pm 12$ & $60 \pm 9$ & $74 \pm 10$ \\
\hline Gender ( $\%$ Male $)$ & $83 \%$ & $67 \%$ & $43 \%$ & $57 \%$ \\
\hline NYHA (I/II/III/IV) & $0 / 28 / 6 / 2$ & $0 / 6 / 0 / 0$ & $0 / 5 / 2 / 0$ & \\
\hline LVEDVI (mL/m²) & $90 \pm 42$ & $58 \pm 26$ & $74 \pm 35$ & \\
\hline LVESVI $\left(\mathrm{mL} / \mathrm{m}^{2}\right)$ & $56 \pm 36$ & $23 \pm 11$ & $42 \pm 25$ & \\
\hline $\operatorname{LVEF}(\%)$ & $38 \pm 13$ & $59 \pm 4$ & $46 \pm 15$ & \\
\hline LVEDP (mmHg) & $9.1 \pm 7.5$ & $21.0 \pm 3.0$ & $17.0 \pm 7.6$ & \\
\hline PCW (mmHg) & $7.6 \pm 7.0$ & $15.0 \pm 5.0$ & $9.6 \pm 6.2$ & \\
\hline $\mathrm{CI}\left(\mathrm{L} / \mathrm{min} / \mathrm{m}^{2}\right)$ & $8.0 \pm 6.9$ & $4.0 \pm 0.9$ & $9.9 \pm 11.0$ & \\
\hline $\mathrm{BNP}(\mathrm{pg} / \mathrm{mL})$ & $303 \pm 450$ & $223 \pm 243$ & $454 \pm 417$ & \\
\hline
\end{tabular}

NYHA; New York Heart Association classification, LVEDVI; left ventricular end-diastolic volume index, LVESVI; left ventricular end-systolic volume index, LVEF; left ventricular ejection fraction, LVEDP; left ventricular end-diastolic pressure, PCW; pulmonary capillary wedge pressure, CI; cardiac index, BNP; brain natriuretic peptide.

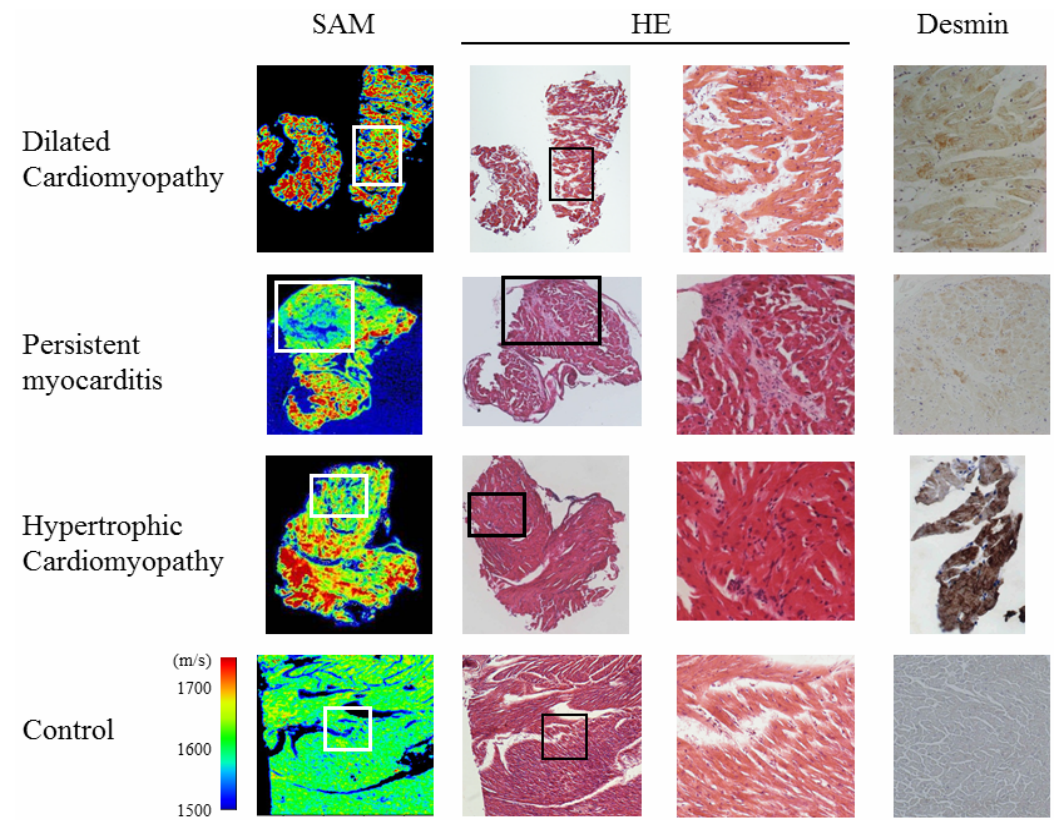

Figure 1. Representative images of each group. Left panel demonstrates the sound speed of myocytes as a color map. Neighboring panels represent the same samples stained with hematoxylin and eosin after measuring sound speed in the tissue. Left panel of HE stain is a close up of the square area of SAM and HE. Right panel indicates immunohistochemical stain using anti-desmin antiserum as a primary antibody. Top: Dilated cardiomyopathy group. Sound speed of myocytes is escalated $(1723 \pm 79 \mathrm{~m} / \mathrm{sec})$ although conventional histology demonstrates almost normal findings. Desmin expression score is 3. Second Panels: Persistent myocarditis group. Histology reveals inflammatory cells infiltrated into the interstitium which shows scant replacement fibrosis. Sound speed of myocytes is moderately escalated $(1669 \pm 66 \mathrm{~m} / \mathrm{sec})$. Third Panels: hypertrophic cardiomyopathy. Sound speed of myocytes escalated to $1695 \pm 56 \mathrm{~m} / \mathrm{sec}$. Disarray is present in the square. Desmin expression score is 4. Bottom Panels: Control myocardium. Sound speed of myocytes is $1613 \pm 12 \mathrm{~m} / \mathrm{sec}$. Histology is completely normal, and no desmin is expressed. Scale bar beside bottom panels relates sound speed to image color. SAM; scanning acoustic microscope. HE; hematoxylin and eosin staining. 
in red in the color image. On the other hand, the sound speed in the persistent myocarditis group was $1673 \pm 30$ $\mathrm{m} / \mathrm{sec}$. This showed a 1.028 -fold escalation of sound speed compared to the controls; however escalated myocytes were sparsely distributed in the tissue. Consequently, there was no significant difference compared to controls.

Finally the myocytes in the hypertrophic cardio-myopathy group also exhibited increased numbers of red myocytes, which showed a striped pattern. The average sound speed was greatest of all here, being $1734 \pm 51$ $\mathrm{m} / \mathrm{sec}$. This was 1.066 -fold cpmpared to controls. Statistically the sound speed in the hypertrophic cardiomyopathy group was significantly greater than that in either the persistent myocarditis group or controls (Figure 2).

\subsection{Desmin Emergence}

Desmin expression was greater in diseased myocytes (cardiomyopathy patients) compared to the controls. Desmin expression score in the hypertrophic cardiomyopathy group was $2.7 \pm 0.8$. It was highest compared to the other diseased groups although there was no significant difference among diseased groups (Figure 3). If patients who did not exhibit desmin emergence were excluded from the cardiomyopathy groups, the sound speed of cardiac myocytes was significantly faster than that of the controls despite the severity of desmin expression (Figure 4).

\section{DISCUSSION}

The present manuscript has demonstrated that the sound speed of myocytes associated with cardiomyopathy was escalated compared to that of normal myocytes. Myocyte sound speed in hypertrophic cardiomyopathy was faster than that in persistent myocarditis, although dilated cardiomyopathy cases demonstrated no significant difference compared to that of persistent myocarditis. Furthermore

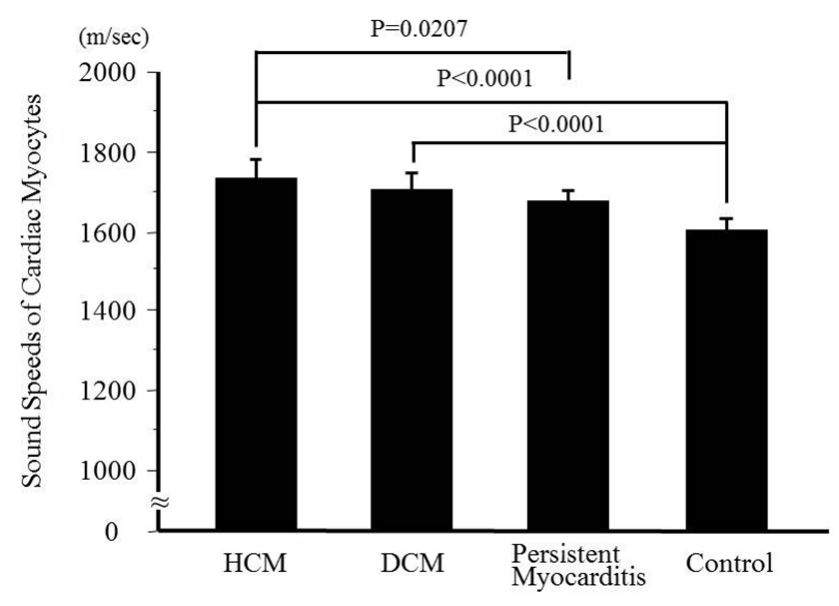

Figure 2. Comparison of sound speed of cardiac myocytes among groups. Cardiomyopathy groups demonstrate increased myocyte sound speed.

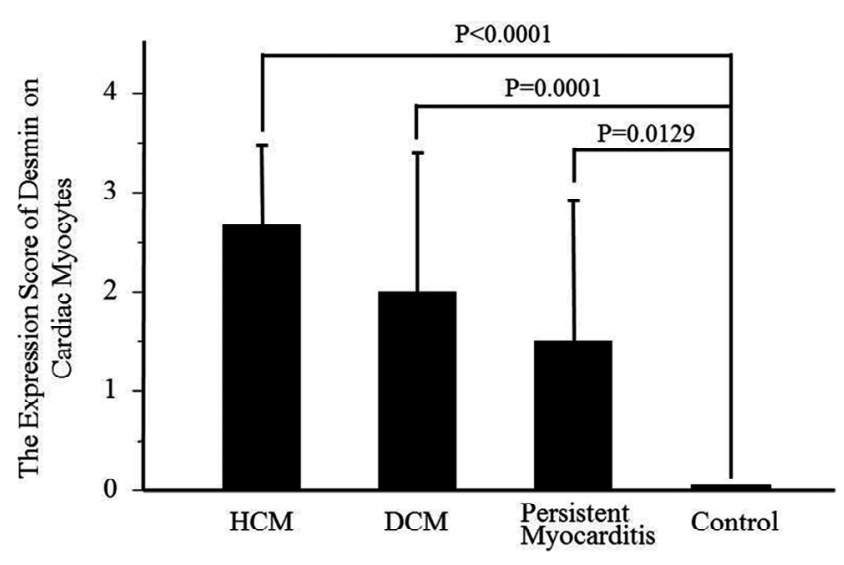

Figure 3. Comparison of desmin expression in cardiac myocytes. Cardiomyopathy groups show greater emergence of desmin in myocytes. 


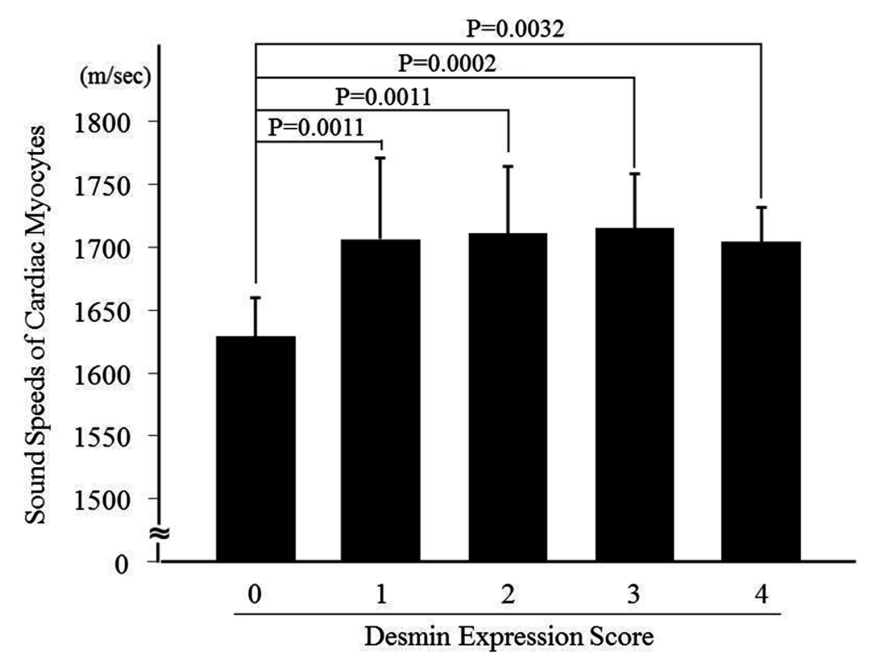

Figure 4. Comparison of sound speed of cardiac myocytes in desmin-expressing cardiomyopathy and controls. The sound speed of cardiac myocytes of desmin expressed cardiomyopathy groups is significantly escalated compared to the controls which desmin expression score was zero.

cardiac myocytes of cardiomyopathy patients exhibited escalated sound speeds if desmin emergence occurred, although the persistent myocarditis group did not exhibit a significant difference compared to the controls, in spite of significantly increased desmin expression score.

Idiopathic dilated cardiomyopathy mainly includes genetic cardiomyopathy and some inflammatory cardiomyopathies [18-20]. In both cases, myocytes show a similar degeneration on conventional histology, despite focal cell infiltration with/without necrosis of myocytes. Furthermore, acquired cardiomyopathy reveals similar phenotypes during the clinical course and in histological findings [6]. This is the principal reason why cardiomyopathy cannot be easily classified according to pathogenesis.

Prochorec-Sobieszek et al. [20] demonstrated that 45\% of dilated cardiomyopathy cases constituted persistent myocarditis, via expression of adhesion molecules and human leukocyte antigen (HLA)-ABC which corresponds to class I molecules of major histocompatibility complex (MHC) in humans and HLA DPQR which belongs to the class II MHC locus, utlizing imunohistological techniques, although conventional optical microscopy identified only $9.5 \%$ of cases. For this reason our dilated cardiomyopathy group may include a number of persistent myocarditis cases. If our dilated cardiomyopathy group could be clearly separated into genetic dilated cardiomyopathy and inflammatory cardiomyopathy groups, the sound speed of myocytes in the dilated cardiomyopathy group might be clearly demonstrated to be faster than that of the persistent myocarditis group. Guidelines of some Associations recommend that 5 to 10 biopsy samples should be obtained to confirm distinctive pathological findings, because diseased lesions are not always included in every specimen [7]. However, we would emphasize that it is sufficient to evaluate the change in myocyte sound speed because some specimens include many myocytes to which are attached abnormal structural cellular proteins in the case of gene-related cardiomyopathy. If the sound speeds of myocytes were too fast, such a sample might include an abnormal cellular protein which would indicate genetic cardiomyopathy; in addition, if the sound speeds of biopsied myocytes indicate mild to moderate escalation, it would be necessary to differentiate acquired cardiomyopathy such as inflammatory cardiomyopathy from idiopathic dilated cardiomyopathy.

Laforsch et al. [21] have reported that acoustic microscopy demonstrated altered elasticity of carapace of Daphnia without major structural change, when guarding itself against assault by an enemy. The sound speed is a cell elasticity marker regardless of the presence or absence of major histological change. This specific marker has not been used to focus on classification of cardiomyopathy, because pathological assessment depends on morphological features, involving immunohistochemistry and molecular biological manipulations of the preparations [22]. Since it had not previously been possible to visualize sound speed, this modality has not been discussed in the assessment of cardiomyopathy. The present manuscript demonstrates that it was possible to evaluate cell sound speed even if samples were embedded in a paraffin block. We had confirmed that the sound speed of frozen myocytes was $1524 \pm 43 \mathrm{~m} / \mathrm{sec}$, and that there-after the same sample exhibited an almost equal sound speed $(1512 \pm 14 \mathrm{~m} / \mathrm{sec})$ after fixation in $10 \%$ formaldehyde. 
However, sound speed of the same myocytes escalated to $1620 \pm 182 \mathrm{~m} / \mathrm{sec}$ after paraffin embedding (unpublished data). From those data, fresh samples are also more appropriate for evaluation of true cell sound speeds, although care needs to be taken to protect the tissue from desiccation. In addition, if samples are embedded in a paraffin block, they need to be compared with normal samples treated with the same procedure through fixation, dehydration and embedding in paraffin. The present acoustic microscopy, in which frequency is $80 \mathrm{MHz}$, showed resolution of $15 \mu \mathrm{m}$ in the axial plane and $18 \mu \mathrm{m}$ in the lateral plane. Normal adult cardiac myocytes are approximately $20 \mu \mathrm{m}$ in width. In the case of cardiomyopathy, cardiac myocytes often show expansion to over $50 \mu \mathrm{m}$ in width. The presently employed acoustic microscopy can clearly separate and assess sound speed of each myocyte.

Desmin, which belongs to the class of intermediate filaments, is a key protein of the cell cytoskeleton, because it connects the contractile apparatus with other structural elements. In the present study, desmin expression was increased in the diseased group with escalation of myocyte sound speed. Also, it was demonstrated that idiopathic cardiomyopathy resulting from abnormalities in cell component proteins escalated the sound speed of myocytes. On the other hand inflammatory cardio-myopathy revealed no significant increase in sound speed if emergence of desmin occurred, because expression of basic intra-cellular proteins may have been conserved. According to the extent of intra-cellular degeneration of myocytes, cell density may have been modified and sound speed might thereby be changed.

Many clinical researchers have sought to evaluate cardiac tissue characterization utilizing integrated backscatter. Several reports have demonstrated diminished cyclic variation reflecting fibrosis of myocardium in dilated cardiomyopathy [23-25]. On the other hand, there are some reports showing that cyclic variation was improved by several therapies and discussing the diminution of cardiac fibrosis [26-29]. Naito et al. [30] reported that cyclic variation had never correlated with cardiac fibrosis in patients with dilated cardiomyopathy. It is reasonable to conclude that cyclic variation improved because sound speeds of myocytes were normalized or restored, and not because cardiac fibrosis was diminished. The present study succeeded in demonstrating escalation of cell sound speed in cardiomyopathy. Diseased myocytes in gene-related cardiomyopathy may not be able to achieve complete contraction or relaxation during cardiac cycles because they have accumulated incomplete functional proteins. Unfortunately, we could not show a change of sound speed in myocytes during cardiac contraction to peak systole and relaxation at end of diastole. Diminished cyclic variation may perhaps depend on less change in sound speed during a cardiac cycle because of incomeplete protein content of myocytes. Recently, several researchers have focused on sound speed of living cells and sought to utilize this technology in several clinical fields including in vivo tissues and cultured cells [31-33]. If modern technology allows development of the acoustic microscope system for use in a catheter-based system such as intracardiac ultrasound, new knowledge may be obtained regarding cyclic variation of myocyte sound speed, leading to diagnosis of genetic cardiomyopathy in vivo.

In conclusion, idiopathic cardiomyopathy (excluding inflammatory cardiomyopathy) escalated the sound speed of myocytes associated with desmin emergence. The sound speed of myocytes has the potential to be used in diagnosis of genetic cardiomyopathy.

\section{LIMITATION}

The present study included the dehydration process to make paraffin-embedded samples. This process influences cell elasticity because of loss of water; furthermore sound speed of target cells should be compared to normal samples treated with the same dehydration and paraffin-embedding procedure.

\section{ACKNOWLEDGEMENTS}

We are especially grateful for the technical support of Miss K. Akazawa (Department of Cardiovascular Medicine, Okayama University Graduate School of Medicine, Dentistry and Pharmaceutical Sciences) in the performance of these experiments.

\section{REFERENCES}

[1] Hershberger, R.E. and Siegfried, J.D. (2011) Update 2011: Clinical and genetic issues in familial dilated cardiomyopathy. Journal of the American College of Cardiology, 57, 1641-1649. doi:10.1016/j.jacc.2011.01.015

[2] Watkins, H., Ashrafian, H. and Redwood, C. (2011) Inherited cardiomyopathies. The New England Journal of Medicine, 364, 1643-1656. doi:10.1056/NEJMra0902923

[3] Marston, S.B. (2011) How do mutations in contractile proteins cause the primary familial cardiomyopathies? Journal of Cardiovascular Translational Research, 4, 245-255. doi:10.1007/s12265-011-9266-2

[4] Theis, J.L., Bos, J.M., Theis, J.D., et al. (2009) Expression patterns of cardiac myofillament proteins-genomic and protein analysis of surgical myectomy tissue from patients with obstructive hypertrophic cardiomyopathy. Circulation: Heart Failure, 2, 325-333. doi:10.1161/CIRCHEARTFAILURE.108.789735

[5] Maron, B.J., Towbin, J.A., Thiene, G., et al. (2006) Contemporary definitins and classification of the cardiomyopathies: An American Heart Association scientific statement from the Council on Clinical Cardiology, Heart Failure and Transplantation Committee; Quality of Care 
and Outcomes Research and Functional Genomics and Translational Biology Interdisciplinary Working Groups; and Council on Epidemiology and Prevention. Circulation, 113, 1807-1816.

doi:10.1161/CIRCULATIONAHA.106.174287

[6] Elliot, P., Andersson, B., Arbustini, E., et al. (2008) Classification of the cardiomyopathies: A position statement from the European society of cardiology working group on myocardial and pericardial diseases. European Heart Journal, 29, 270-276. doi:10.1093/eurheartj/ehm342

[7] Cooper, L.T., Baughman, K.L., Feldman, A.M., et al. (2007) The role of endomyocardial biopsy in the management of cardiovascular disease: A scientific statement from the American heart association, the American college of cardiology, and the European society of cardiology. Circulation, 116, 2216-2233. doi:10.1161/CIRCULATIONAHA.107.186093

[8] Hauck, A.J., Kearney, D.L. and Edwards, W.D. (1989) Evaluation of postmortem endomyocardial biopsy specimens from 38 patients with lymphocytic myocarditis: Implications for role of sampling error. Mayo Clinic Proceedings, 64, 1235-1245. doi:10.1016/S0025-6196(12)61286-5

[9] Chow, L.H., Radio, S.J., Sears, T.D., et al. (1989) Insensitivity of right ventricular endomyocardial biopsy in the diagnosis of myocarditis. Journal of the American College of Cardiology, 14, 915-920. doi:10.1016/0735-1097(89)90465-8

[10] Chow, L.C., Dittrich, H.C. and Shabetai, R. (1988) Endomyocardial biopsy in patients with unexplained congestive heart failure. Annals of Internal Medicine, 109, 535-539.

[11] Sokolov, S. (1949) The ultrasonic microscope. Doklandy Akademii Nauk SSSR, 64, 333-335.

[12] Lemons, R.A. and Quate, C.F. (1975) Acoustic microscopy: Biomedical applications. Science, 188, 905-911.

[13] Saijo, Y., Sasaki, H., Sato, M., et al. (2000) Visualization of human umbilical vein endothelial cells by acoustic microscopy. Ultrasonics, 38, 396-399. doi:10.1016/S0041-624X(99)00200-0

[14] Saijo, Y., Jorgensen, C.S. and Falk, E. (2001) Ultrasonic tissue characterization of collagen in lipid-rich plaques in apo E-deficient mice. Atherosclerosis, 158, 289-295. doi:10.1016/S0021-9150(01)00435-X

[15] Okawai, H., Kobayashi, K. and Nitta, S. (2001) An approach to acoustic properties of biological tissues using acoustic micrographs of attenuation constant and sound speed. Journal of Ultrasound in Medicine, 20, 891-907.

[16] Hozumi, N., Yamashita, R., Lee, C.K., et al. (2004) Timefrequency analysis for pulse driven ultrasounic microscopy for biologicaltissue characterization. Ultrasonics, 42, 717-722. doi:10.1016/j.ultras.2003.11.005

[17] Hagiwara, Y., Saijo, Y., Chimoto, E., et al. (2006) Increased elasticity of capsule after immobilization in a rat knee experimental model assessed by scanning acoustic microscopy. Upsala Journal of Medical Sciences, 111, 303-313. doi:10.3109/2000-1967-054

[18] Towbin, J.A., Lowe, A.M., Colan, S.D., et al. (2006) In- cidence, causes, and outcomes of dilated cardiomyopathy in children. JAMA, 296, 1867-1876. doi:10.1001/jama.296.15.1867

[19] Baughman, K.L. (2006) Diagnosis of myocarditis: Death of Dallas criteria. Circulation, 113, 593-595. doi:10.1161/CIRCULATIONAHA.105.589663

[20] Prochorec-Sobieszek, M., Bilinska, Z.T., Grzybowski, J., et al. (2006) Assessment of the inflammatory process by endomyocardial biopsy in patinets with dilated cardiomyopathy based on pathological and immunohistochemical methods. Kardiologia Polska, 64, 479-487.

[21] Laforsch, C., Ngwa, W., Grill, W., et al. (2004) An acoustic microscopy technique reveals hidden morphological defenses in Daphnia. PNAS, 101, 15911-15914. doi:10.1073/pnas.0404860101

[22] Leone, O., Veinot, J.P., Angelini, A., et al. (2012) 2011 consensus statement on endomyocardial biopsy from the association for European cardivascular pathology and the society for cardiovascular pathology. Cardiovascular $\mathrm{Pa}$ thology, 21, 245-274. doi:10.1016/i.carpath.2011.10.001

[23] Fujimoto, S., Mizuno, R., Nakagawa, Y., et al. (1999) U1trasonic tissue characterization in patients with dilated cardiomyopathy: Comparison with findings from right ventricular endomyocardial biopsy. International Journal of Cardiac Imaging, 15, 391-396. doi:10.1023/A:1006272919061

[24] Di Bello, V., Giorgi, D., Viacava, P., et al. (2004) Severe aortic stenosis and myocardial function: Diagnostic and prognostic usefulness of ultrasonic integrated backscatter analysis. Circulation, 110, 849-855. doi:10.1161/01.CIR.0000138930.12773.41

[25] Mor Avi, V., Lang, R.M., Badano, L.P., et al. (2011) Current and evolving echocardigraphic techniques for the quantitative evaluation of cardiac mechanics: ASE/EAE consensus statement on methodology and indications endorsed by the Japanese society of echocardiography. Journal of the American Society of Echocardiography, 24, 277-313. doi:10.1016/j.echo.2011.01.015

[26] Suwa, M., Ito, T., Kobashi, A., et al. (2000) Myocardial integrated ultrasonic backscatter in patients with dilated cardiomyopathy: Prediction of response to beta-blocker therapy. American Heart Journal, 139, 905-912. doi:10.1016/S0002-8703(00)90024-3

[27] Lin, Y.H., Lee, H.H., Liu, K.L., et al. (2011) Reversal of myocardial fibrosis in patients with unilateral hyperaldosteronism receiving adrenalectomy. Surgery, 150, 526533. doi:10.1016/j.surg.2011.02.006

[28] Mizuta, Y., Kai, H., Mizoguchi, M., et al. (2008) Longterm treatment with Valsartan improved cyclic variation of the myocardial integral backscatter signal and diastolic dysfunction in hypertensive patients: The echocardiographic assessment. Hypertension Research, 31, 18351842. doi:10.1291/hypres.31.1835

[29] Fijalkowski, M., Koprowski, A., Galaska, R., et al. (2010) Improvement of ultrasonic myocardial properties after aortic valve replacement for pure severe aortic stenosis: The predictive value of ultrasonic tissue characterization for left ventricle reverse remodeling. Journal of the American Society of Echocardiography, 23, 1060-1066. 
doi:10.1016/j.echo.2010.07.018

[30] Naito, J., Masuyama, T., Mano, T., et al. (1996) Ultrasonic myocardial tissue characterization in patients with dilated cardiomyopathy: Value in noninvasive assessment of myocardial fibrosis. American Heart Journal, 131, 115-121. doi:10.1016/S0002-8703(96)90059-9

[31] Weiss, E.C., Lemor, R.M., Pilarczyk, G., et al. (2007) Imaginf of focal contacts of chicken heart muscle cells by high-frequency acoustic microscopy. Ultrasound in Medicine and Biology, 33, 1320-1326. doi:10.1016/j.ultrasmedbio.2007.01.016

[32] Hagiwara, Y., Saijo, Y., Ando, A., et al. (2009) Ultrasonic intensity microscopy for imaging of living cells. Ultrasonics, 49, 386-388.

doi:10.1016/j.ultras.2008.10.013

[33] Winterroth, F., Lee, J., Kuo, S., et al. (2011) Acoustic microscopy analyses to determine good vs failed tissue engineered oral mucosa under normal or thermally stresssed culture conditions. Annals of Biomedical Engineering, 39, 44-52. doi:10.1007/s10439-010-0176-2 